\title{
Tougu Xiaotong capsule exerts a therapeutic effect on knee osteoarthritis by regulating subchondral bone remodeling
}

\author{
GUANGWEN WU ${ }^{1 *}$, JIAHUI ZHANG $^{1 *}$, WENLIE CHEN $^{1,2}$, SAINAN CHEN $^{1,4}$, YUNMEI HUANG $^{2}$, \\ RUHUI LIN ${ }^{1}$, MEIYA HUANG ${ }^{2},{\text { ZUANFANG } \text { LI }^{2} \text {, LIANGPU ZHENG }}^{3}$ and XIHAI LI ${ }^{3}$ \\ ${ }^{1}$ Academy of Integrative Medicine; ${ }^{2}$ National Laboratory of Traditional Chinese Medicine Pharmacology \\ (Cell Structure and Function); ${ }^{3}$ Fujian Key Laboratory of Integrative Medicine on Geriatrics; \\ ${ }^{4}$ Fujian Academy of Traditional Chinese Medicine, Fujian University of Traditional Chinese Medicine, \\ Fuzhou, Fujian 350122, P.R. China
}

Received November 12,2018; Accepted December 12, 2018

DOI: $10.3892 / \mathrm{mmr} .2018 .9778$

\begin{abstract}
Previous studies have shown that Tougu Xiaotong capsule (TGXTC) has therapeutic effects on knee osteoarthritis (OA) through multiple targets. However, the mechanisms of action underlying its regulation of subchondral bone reconstruction remain unclear. In this study, we investigated the effects of TGXTC on subchondral bone remodeling. Eighteen six-month-old New Zealand white rabbits of average sex were randomly divided into the normal, model and TGXTC groups. The rabbit knee OA model was induced by a modified Hulth's method in the model and TGXTC groups, but not the normal group. Five weeks postoperatively, intragastric administration of TGXTC was performed for four weeks. After drug administration, the medial femoral condyle and tibia were prepared for observation of cartilage histology via optical microscopy and micro-computed tomography, the serum was collected for biochemical parameters assay and the subchondral bone isolated from the lateral femoral condyle was collected for detection of IL-1 $\beta$ and TNF- $\alpha$ mRNA and protein by reverse transcription-quantitative polymerase chain reaction and western blot analysis, respectively. The results showed that treatment with TGXTC significantly mitigated cartilage injury and subchondral bone damage, improved the parameter of subchondral trabecular bone, decreased alkaline phosphatase and tartrate-resistant acid phosphatase activity,
\end{abstract}

Correspondence to: Professor Wenlie Chen or Dr Sainan Chen, Academy of Integrative Medicine, Fujian University of Traditional Chinese Medicine, 1 Qiuyang Road, Fuzhou, Fujian 350122, P.R. China

E-mail: chen.wl@163.com

E-mail: chensainan@fjszyy.com

*Contributed equally

Key words: osteoarthritis, subchondral bone remodeling, micro-CT, trabecular bone parameters, bone formation, bone resorption, Tougu Xiaotong Capsule and significantly reducing the osteoprotegerin/receptor activator of nuclear factor- $\mathrm{\kappa B}$ ligand ratio, reduced the expression of IL- $1 \beta$ and TNF- $\alpha$ mRNA and protein. These results suggest that TGXTC could delay the pathological development of OA by regulating subchondral bone remodeling through regulation of bone formation and bone resorption and its relating inflammatory factors, and this may partly explain its clinical efficacy in the treatment of knee OA.

\section{Introduction}

Osteoarthritis (OA) is characterized by the degradation and loss of articular cartilage, inflammation of the synovial membrane, osteophyte formation and changes in the subchondral bone (1-4). Previous studies focused on cartilage, the subchondral bone was recently found to play a crucial role in OA $(5,6)$. Subchondral bone loss occurs during the early stage of OA while subchondral sclerosis occurs in the late stage of OA, along with the presence of osteophytes (7). In addition, a vicious cycle between structural changes in the subchondral bone and cartilage damage occurs, which contributes to the progression of OA. In a word, the subchondral bone is another interesting intervention point for the treatment of OA.

Therefore, to understand the microstructure of the subchondral bone is very important in OA. Micro-computed tomography (micro-CT) can detect microstructural changes and measure parameters of subchondral bone (8). The most important change in the subchondral bone is the trabecular bone. Therefore, it is necessary to evaluate the structural changes of trabecular bone and its related parameters by micro-CT. Moreover, the parameters and structural changes of subchondral bone are related to bone remodeling $(9,10)$.

Bone remodeling plays an essential role in the balance of formation and resorption in bone tissue (11). In this regard, alkaline phosphatase (ALP) can enhance osteoblast differentiation and promote bone formation $(12,13)$. Tartrateresistant acid phosphatase (TRAP) is used as a marker for osteoclasts and bone resorption (14). The osteoprotegerin $(\mathrm{OPG}) /$ receptor activator of nuclear factor- $\kappa \mathrm{B}$ ligand (RANKL) system is one of the most essential molecular mechanisms to regulate bone remodeling. Imbalances in the OPG/ 
RANKL system can cause abnormal bone metabolism and subchondral bone damage (15-17). The OPG/RANKL ratio is vital to the bone mass because it maintains normal bone turnover $(18,19)$. Previous findings have shown that regulation of bone metabolism occurs through the classical OPG/ RANK/RANKL pathway, the direct and indirect functions of which are influenced by numerous factors, including IL- $1 \beta$ and TNF- $\alpha(20,21)$.

Tougu Xiaotong capsule (TGXTC) is an effective prescription in OA treatment which consists of Ligusticum chuanxiong, Morinda officinalis, Sarcandra glabra and Paeonia lactiflora $(22,23)$. There are many effective molecules in TGXTC, which is multi-drug, multi-path and multi-target of molecular mechanism for the treatment of OA, and the non-linear regulation pattern exists in TGXTC ligand-target interaction network (24). Previous studies have proved that TGXTC has therapeutic effects on knee OA (25) through multiple targets, such as inhibiting chondrocyte apoptosis (26), improving the structure and function of cartilage (27) and slowing down cartilage degradation (23), promoting osteoblast proliferation and calcium secretion (22). However, evaluation of the effect of TGXTC on subchondral bone remodeling using micro-CT combined with markers of bone formation and bone resorption has not been reported.

To further elucidate the precise mechanism of the potential treatment of OA, in the present study, we investigated the effects of TGXTC in the bone remodeling of subchondral bone on the basis of micro-CT assessment of trabecular bone. We found could TGXTC delay the pathological development of OA through regulating formation/resorption balance and it's relating inflammatory factor to improve the remodeling of subchondral bone.

\section{Materials and methods}

Animals. A total of 18 female New Zealand rabbits (age, 6 months; weight, $1.7-2.3 \mathrm{~kg}$ ) were purchased from the Shanghai City Songjiang District Songlian Experimental Animal Farm (Shanghai, China). These animals were bred in the Animal Center of Fujian University of Traditional Chinese Medicine (Fujian, China). The present study was approved by the Animal Care and Use Committee of the Fujian University of Traditional Chinese Medicine (Fujian, China).

Drugs and reagents. TGXTC was prepared by the Second People's Hospital of Fujian University of Traditional Chinese Medicine (Fujian, China) (approval no. MIN ZIZHI Z20100006). Sodium pentobarbital was obtained from ShanghaiXitang Biotechnology Co., Ltd., (Shanghai, China), sodium penicillin was purchased from GE Healthcare Life Sciences (Logan, UT, USA), paraformaldehyde was from Beijing Solarbio Science \& Technology Co., Ltd., (Beijing, China), and EDTA was provided by Sinopharm Chemical Reagent Co.,Ltd. (Shanghai, China). The ALP and tartrate resistant acid phosphatase assay kits were obtained from Beyotime Institute of Biotechnology (Nantong, China). The rabbit OPG ELISA kit and rabbit RANKL ELISA kit were purchased from Shanghai Westang Bio-Tech Co., Ltd. (Shanghai, China). Other reagents included TRIzol, which was obtained from Invitrogen Life Technologies (Carlsbad, CA, USA). PrimeScript ${ }^{\mathrm{TM}}$ RT reagent kit with gDNA Eraser and $\mathrm{SYBR}^{\circledR}$ Premix Ex Taq TM II were purchased from Takara Bio., Inc. (Otsu, Japan), mouse anti- $\beta$-actin antibody (monoclonal; 1:8,000; cat. no. HC201), goat anti-mouse horseradish peroxidase-conjugated IgG (monoclonal; 1:4,000; cat. no. HS201) and goat anti-rabbit horseradish peroxidase-conjugated IgG (monoclonal, 1:4,000; cat. no. HS101) were purchased from TransGen Biotech Co., Ltd., (Beijing, China), rabbit anti-IL-1 $\beta$ antibody (polyclonal; 1:500; cat. no. 16806-1-AP) was provided by Wuhan Sanying Biotechnology (Hubei, China), and rabbit anti-TNF- $\alpha$ (monoclonal; 1:500; cat. no. MAB2103) antibody was obtained from R\&D Systems China (Shanghai, China). All other chemicals, unless otherwise stated, were obtained from Sigma-Aldrich (Merck KGaA, St. Louis, MO, USA).

Grouping, model preparation and treatments. The 18 rabbits were randomly divided into three groups, including the normal, model and TGXTC group, with 6 rabbits in each group.

The rabbit OA model was established by using modified Hulth's method in all groups except for the normal group (28). Rabbits were anesthetized by ear vein injection with sodium pentobarbital $(30 \mathrm{mg} / \mathrm{kg})$. After a routine disinfection, the medial collateral and anterior cruciate ligaments were transected via the medial approach, and the medial meniscus was excised. Then the joint capsule was sutured layer by layer. Prophylactic antibiotic with sodium penicillin (400,000 units) was given for three days after surgery. After a week, the animals were forced to movement for $30 \mathrm{~min}$ daily for one month.

After five weeks, a clinical oral dose of TGXTC (140 mg/kg/day) was given to rabbits in the TGXTC group for four consecutive weeks. An equivalent volume of saline was administered to the normal and model group rabbits.

Specimen collection. After intragastric drug administration, the rabbits were sacrificed by air embolism after anesthesia with $3 \%$ sodium pentobarbital $(30 \mathrm{mg} / \mathrm{kg}$ ear marginal vein injection). The femur, tibia and serum were harvested. The medial femoral condyle was prepared for Safranin O-fast green staining and the tibia for micro-CT, respectively. The serum was collected for biochemical parameters assay. The subchondral bone isolated from the lateral femoral condyle was collected for reverse transcription-quantitative polymerase chain reaction (RT-qPCR) and western blot analysis.

Histology. The medial femoral condyle tissues were fixed in $4 \%$ paraformaldehyde for 3 days and decalcified in 10\% EDTA at room temperature for about 4 months. The medial femoral condyle was longitudinally cut and embedded in paraffin. Sagittal sections $(4 \mu \mathrm{m})$ were prepared for Safranin O-fast green staining and observed under an optical microscope (DM4000 B; Leica Microsystems GmbH, Wetzlar, Germany) and images were captured at a magnification, x100. Following that a modified Mankin scoring principles (29) was used to evaluate the degeneration of each femoral condyle.

Micro-CT evaluation. A micro-CT system (ZKKS-MCT; Zhongke Kaisheng Medical Technology Co., Ltd., Guangzhou, China), with a source voltage of $60 \mathrm{kV}$, a power of $40 \mathrm{~W}$, a current of $666 \mu \mathrm{A}$, and resolution of $50 \mu \mathrm{m}$, was used to obtain 
$\mathrm{X}$-ray radiographs. The specimens were attached to a stage that rotated $360^{\circ}$ with images captured every $0.72^{\circ}$. A 3D analysis was used to calculate parameters of the tibial in the region at $4.2 \times 1.2 \times 1.8 \mathrm{~mm}$ from the subchondral bone. The parameters measured were: Tabecular number (Tb.N), trabecular thickness (Tb.Th), bone volume fraction (BV/TV) and trabecular separation (Tb.Sp).

Serum biochemistry assay. After treatment, blood samples were collected and centrifuged at $3,000 \mathrm{x}$ g for $10 \mathrm{~min}$ at $4^{\circ} \mathrm{C}$. The supernatants were stored at $-20^{\circ} \mathrm{C}$ until analysis. ALP and TRAP activity was measured with autobiochemical analyzer by standard colorimetric methods using detection kits, according to the manufacturer's protocol. Serum OPG and RANKL levels were observed by ELISA kit, according to the manufacturer's protocol.

$R T-q P C R$. Total RNA was extracted from the subchondral bone of the lateral femoral condyle using TRIzol and quantified using a BioPhotometer Plus UV spectrophotometer (Eppendorf AG, Hamburg, Germany). cDNA was synthesized using a Takara PrimeScript $^{\text {TM }}$ RT reagent kit with gDNA Eraser. Primers were designed and produced by Takara Bio., Inc. (Table I). The PCR system was prepared according to the manufacturer's instructions, with $10 \mu \mathrm{l}$ of SYBR ${ }^{\circledR}$ Premix Ex Taq II, $0.4 \mu 1$ of ROX Reference Dye II, $0.8 \mu \mathrm{l}$ of upstream primer, $0.8 \mu \mathrm{l}$ of downstream primer, $2 \mu \mathrm{l}$ of cDNA, and $6 \mu \mathrm{l}$ of $\mathrm{dH} 2 \mathrm{O}, 20 \mu \mathrm{l}$ in total. The PCR amplification protocol was: Pre-denaturation at $95^{\circ} \mathrm{C}$ for $30 \mathrm{sec}$, denaturation at $95^{\circ} \mathrm{C}$ for $3 \mathrm{sec}$, and annealing at $60^{\circ} \mathrm{C}$ for $30 \mathrm{sec}$, for a total of 40 cycles. The fluorescence signal of GAPDH acted as an internal reference for calculating the relative gene expression levels.

Western blot analysis. The subchondral bone isolated from the lateral femoral condyle was immersed 1:10 in lysis buffer containing 50mM Tris ( $\mathrm{pH} 7.4$ ), $150 \mathrm{mM} \mathrm{NaCl}, 1 \%$ Triton $\mathrm{X}-100,1 \%$ sodium deoxycholate, $0.1 \%$ SDS, $0.1 \%$ sodium orthovanadate and $2 \mathrm{mM}$ EDTA (Beijing BLKW Biotechnology Co., Ltd., Shanghai, China), homogenized using a Tissuelyser-192 (Shanghai Jingxin Industrial Development Co., Ltd., Shanghai, China) on ice, then centrifuged at $4^{\circ} \mathrm{C}$ at $12,000 \mathrm{x} g$ for $30 \mathrm{~min}$. A bicinchoninic acid kit (cat. no. P0010; Beyotime Institute of Biotechnology) was applied to determine protein concentrations. Protein samples $(30 \mu \mathrm{g})$ were electrophoresed by $12 \%$ sodium dodecyl sulfate polyacrylamide gel electrophoresis for $2 \mathrm{~h}$ (Beyotime Institute of Biotechnology, Shanghai, China), transferred onto polyvinylidene difluoride (Shanghai Jinghong Laboratory Instrument Co., Ltd.) membranes, and blocked with 5\% skimmed milk for $2 \mathrm{~h}$. Next, the samples were incubated on a shaker at $4{ }^{\circ} \mathrm{C}$ overnight with primary antibodies against IL- $\beta$ (1:500), TNF- $\alpha$ (1:500) and $\beta$-actin $(1: 8,000)$. The samples were then rinsed with TBST, incubated with their corresponding secondary antibodies: Goat anti-mouse horseradish peroxidase-conjugated IgG (monoclonal; 1:4,000; cat. no. HS201) or goat anti-rabbit horseradish peroxidase-conjugated IgG (monoclonal, 1:4,000; cat. no. HS101) both from TransGen Biotech, Inc., on a shaker at room temperature for $1 \mathrm{~h}$, rinsed with TBST, and developed using ECL substrate. Image processing was carried out using the ChemiDoc ${ }^{\mathrm{TM}}$ XRS + system (Bio-Rad Laboratories, Inc.,
Table I. Primers.

Product

\begin{tabular}{llc} 
Genes & \multicolumn{1}{c}{ Sequence } & length (bp) \\
\hline IL-1 $\beta$ & F: 5'-ACAAGTGGTGT & 125 \\
& TCTCCATGAGTTT-3' & \\
& R: 5'-GGGTAGGTTTA & \\
& TCGTCTTTCATCAC-3' & \\
TNF- $\alpha$ & F: 5'-ACGTAGTAGCA & 150 \\
& AACCCGCAAG-3' & \\
& R: 5'-TGAAGAGAACC & \\
& TGGGAGTAGATGAG-3' & \\
GAPDH & F: CCACTTTGTGA & \\
& AGCTCATTTCT -3' & \\
& F:5'-TCGTCCTCCTC \\
& TGGTGCTCT-3'
\end{tabular}

Hercules, CA, USA) with BeyoECL Plus (Beyotime Institute of Biotechnology) to analyze gray values and to determine the relative expression of the proteins.

Statistical analysis. Data were expressed as mean \pm standard deviation and were analyzed by SPSS version 20.0 software. The Shapiro-Wilk test was used to determine the normality of all groups of data. If the data exhibited a normal distribution, they were analyzed with one-way analysis of variance (ANOVA) followed by least signifcant difference or Games Howell post hoc tests. If not, the Kruskal-Wallis test was used and the Mann-Whitney U with Bonferroni's correction was applied as a post hoc test. $\mathrm{P}<0.05$ was considered to indicate a statistically signifcant difference.

\section{Results}

TGXTC decreased cartilage damage. To determine the effects of TGXTC on cartilage morphology, the sections were stained with Safranin O-fast green and observed under an optical microscope. As shown in Fig. 1A, the superficial zone, mid zone, deep zone, and calcified cartilage were visible; the surface of articular cartilage was smooth and had no cracks, the chondrocytes were arranged in rows, and the tidemark and cement line were clear. The proteoglycans were stained by Safranin O, and fast green staining was evident in the superficial zone and the subchondral bone in the normal group. In the model group, the cartilage surface was damaged with cracks and disordered chondrocyte clusters, and the tidemark replication. In addition, staining of proteoglycans was severely reduced (Fig. 1B). However, following treatment with TGXTC, the articular cartilage had little rough surface without cracks, chondrocytes arrangement were arranged in order, and the cartilage matrix stained became deeper and even better than the normal group (Fig. 1C).

We used the scoring principles of Mankin to analyze the change of cartilage degeneration. The Mankin score in the model group was significantly higher compared with the normal group $(\mathrm{P}<0.01)$. However, the Mankin score was 

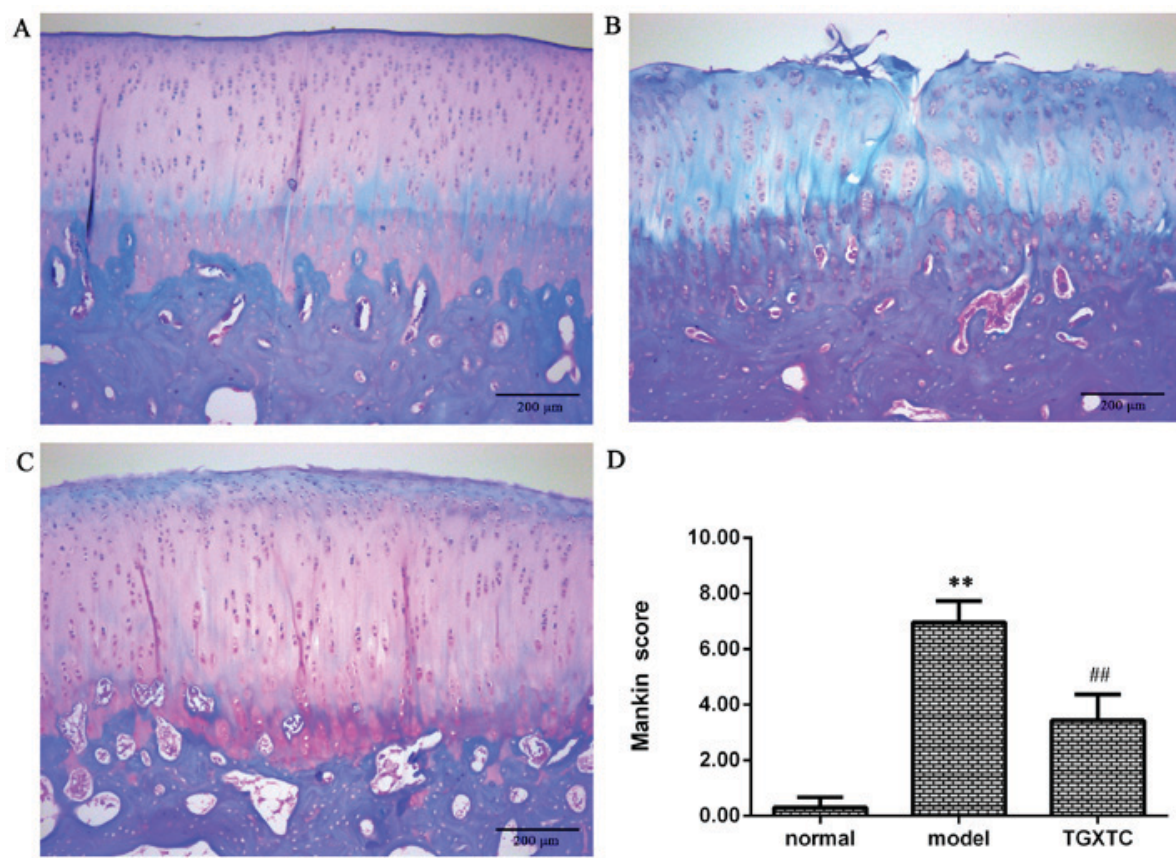

Figure 1. Effect of TGXTC on the structure of cartilage. (A) Normal. (B) Model. (C) TGXTC groups. (D) Mankin score. Magnification, x100; scale bar, $200 \mu \mathrm{m}$. Values are presented as the mean \pm standard deviation. ${ }^{* *} \mathrm{P}<0.01$ vs. normal group; ${ }^{\# \#} \mathrm{P}<0.01$ vs. model group; TGXTC, Tougu Xiaotong capsule.
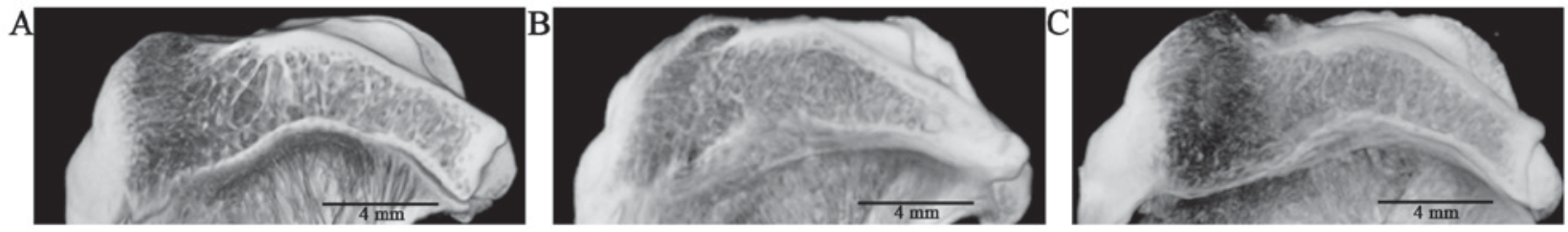

Figure 2. Effect of TGXTC on the structural change of sagittal plane. (A) Normal. (B) Model. (C) TGXTC groups. Scale bar, 4 mm. TGXTC, Tougu Xiaotong capsule.
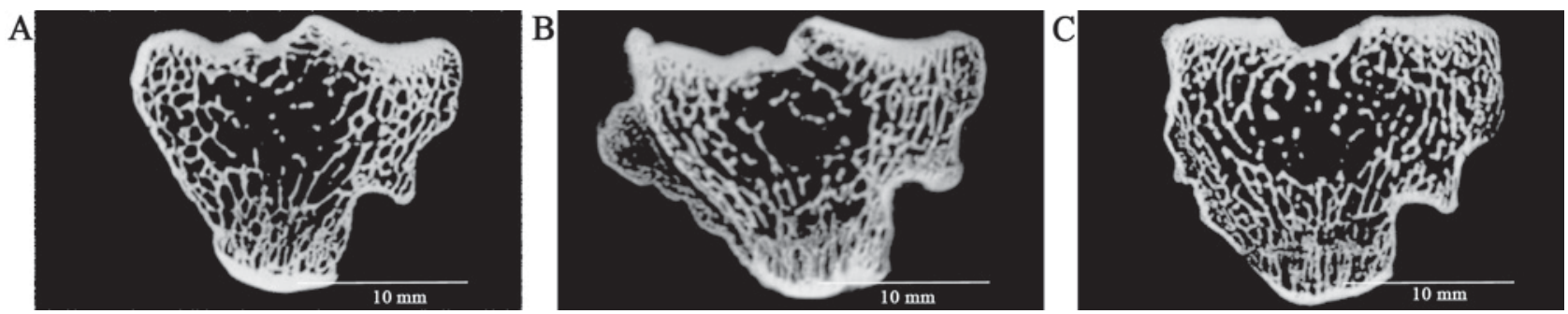

Figure 3. Effect of TGXTC on the cross-sectional change. (A) Normal. (B) model. (C) TGXTC groups. Scale bar, 10 mm. TGXTC, Tougu Xiaotong capsule.

significantly lower in the TGXTC group than in the model group $(\mathrm{P}<0.01)$. According to the Mankin score, $1-5$ constitutes early stage of OA; 6-9, middle stage of OA; and 10-14, late stage of OA. The results suggest that the cartilage damage in the model group was in the middle stages of OA, while for the TGXTC group cartilage damage occurred in the early stages of OA (Fig. 1D).

TGXTC reversed microstructural damage of subchondral bone. In the sagittal plane of the subchondral bone, the trabecular bone was arranged in an orderly manner in the normal group (Fig. 2A). In the model group, the trabecular bone became broken and disordered (Fig. 2B). However, after treatment with TGXTC, trabecular bone was relatively regular (Fig. 2C).

In the cross section of subchondral bone, the trabecular bone had a uniform network in the normal group (Fig. 3A). In the model group, increased disorder of the trabecular bone was evident with a large osteophyte (Fig. 3B). However, after treatment, the structure of trabecular bone was improved and arranged neatly (Fig. 3C).

In the 3D models of each experimental group, tibial plateau became deformed with large osteophyte in the model group (Fig. 4B), while no osteophyte was present in the normal group (Fig. 4A). In the TGXTC group, the osteophyte was significantly decreased compared to the model group (Fig. 4C). 

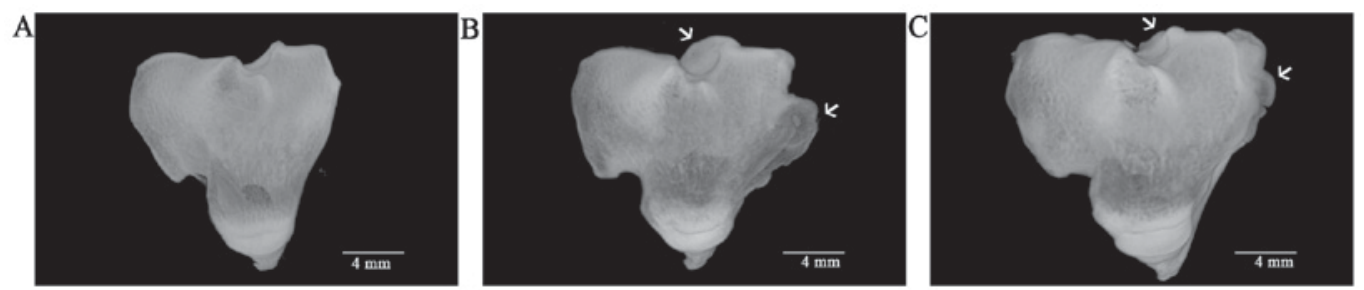

Figure 4. Effect of TGXTC on the micro-CT 3D models of tibial plateau. (A) Normal. (B) Model. (C) TGXTC groups. Arrows indicate osteophyte. Scale bar, $4 \mathrm{~mm}$. TGXTC, Tougu Xiaotong capsule.

A

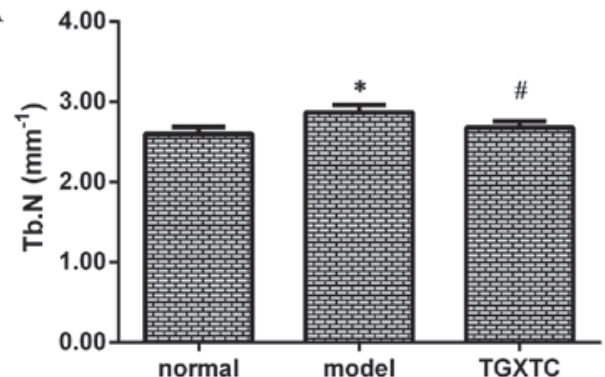

$\mathrm{C}$

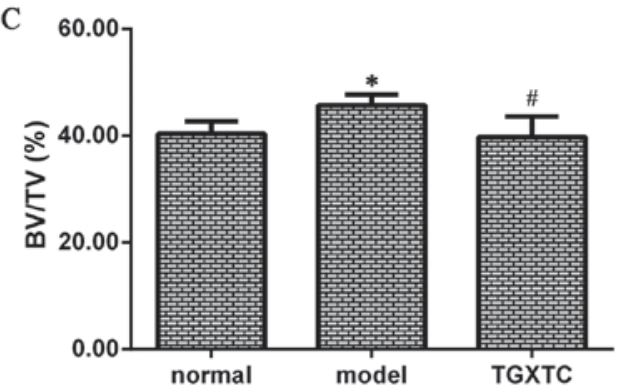

B

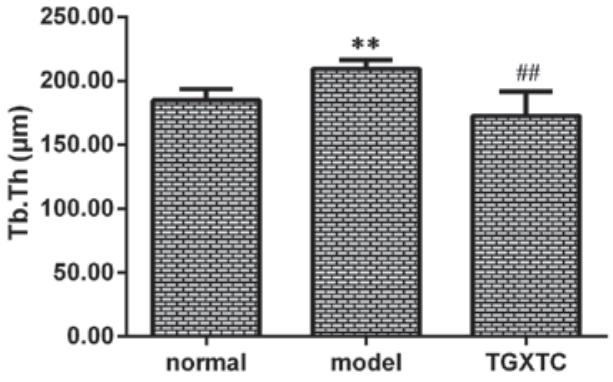

D

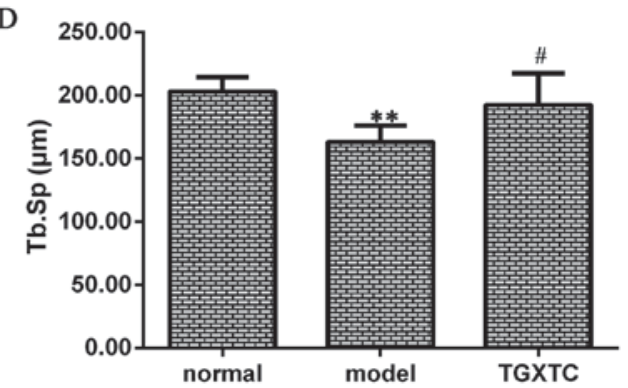

Figure 5. Effect of TGXTC on the parameter of subchondral trabecular bone. Values are presented as the mean \pm standard deviation. (A) Trabecular number (Tb.N). (B) Trabecular thickness (Tb.Th). (C) Bone volume fraction (BV/TV). (D) Trabecular separation (Tb.Sp). "P<0.05; "* $\mathrm{P}<0.01$ vs. normal group; "P<0.05; ${ }^{\#} \mathrm{P}<0.01$ vs. model group. TGXTC, Tougu Xiaotong capsule.

A

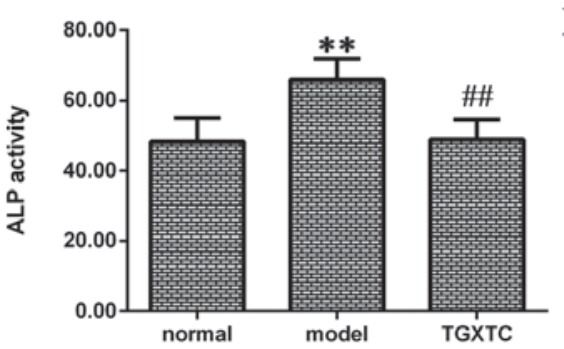

B

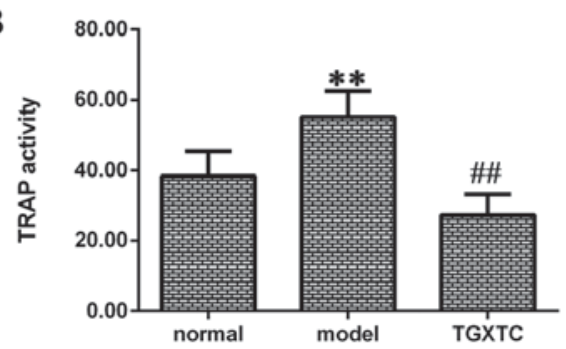

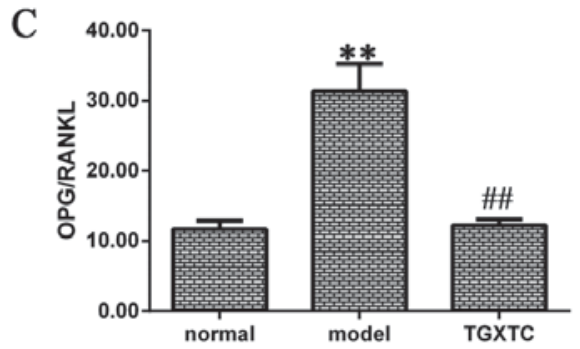

Figure 6. Effect of TGXTC on serum ALP and TRAP and OPG/RANKL ratio. (A) ALP activity. Values are presented as the mean \pm standard deviation. (B) TRAP activity. (C) OPG/RANKL ratio. ${ }^{* *} \mathrm{P}<0.01$ vs. normal group; ${ }^{\# \#} \mathrm{P}<0.01$ vs. model group.

The mean and standard deviation values of the micro-CT parameters are shown in Fig. 5. The Tb.N, Tb.Th and BV/TV were increased while the Tb.Sp was decreased in the model group compared with the normal group $(\mathrm{P}<0.05$ or $\mathrm{P}<0.01)$. However, Tb.N, Tb.Th and BV/TV were reduced in the TGXTC compared with the model group, whereas Tb.Sp in the TGXTC was increased compared to the model group $(\mathrm{P}<0.05$ or $\mathrm{P}<0.01)$. These results suggest that subchondral sclerosis and microstructure damage in the model group, whereas TGXTC could improve structural injury of subchondral bone.
TGXTC decreased ALP and TRAP. ALP activity was significantly higher in the model than the normal group $(\mathrm{P}<0.01$; Fig. 6A). After treatment, the TGXTC group exhibited a significant decrease in the ALP activity compared with the model group $(\mathrm{P}<0.01$; Fig. $6 \mathrm{~A})$.

A similar trend was identified for TRAP activity (Fig. 6B). After inducing OA, TRAP activity was significantly higher in the model compared to the normal group $(\mathrm{P}<0.01)$. After treatment, the TGXTC group exhibited a significant decrease in TRAP activity compared to the model group $(\mathrm{P}<0.01)$. 

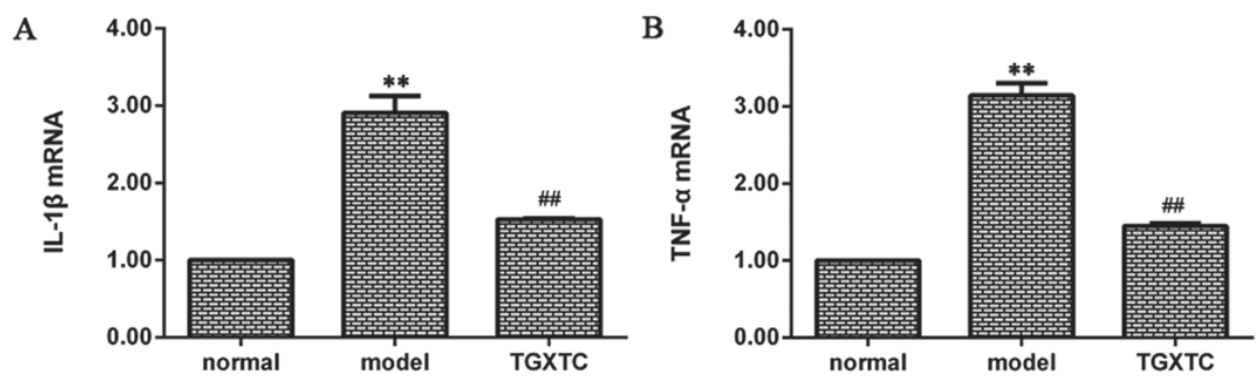

Figure 7. Effects of TGXTC on the mRNA expression of IL-1 $\beta$ and TNF- $\alpha$. (A) The relative mRNA expression of IL-1 $\beta$. (B) The relative mRNA expression of TNF- $\alpha$. Values are presented as the mean \pm standard deviation. ${ }^{* *} \mathrm{P}<0.01$ vs. normal group; ${ }^{\# \#} \mathrm{P}<0.01$ vs. model group. TGXTC, Tougu Xiaotong capsule.

A
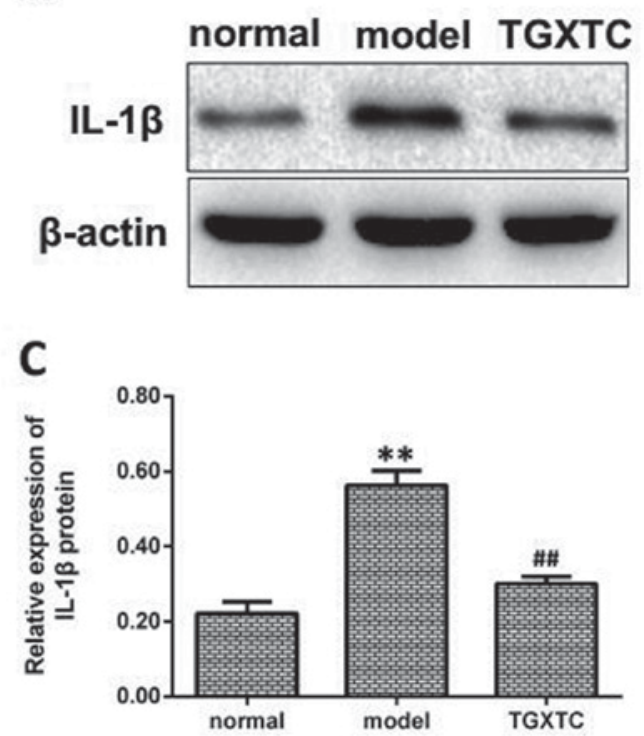

B

\section{normal model TGXTC}

TNF-c

$\beta$-actin

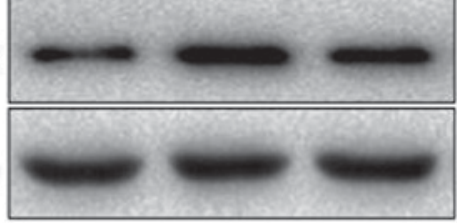

D

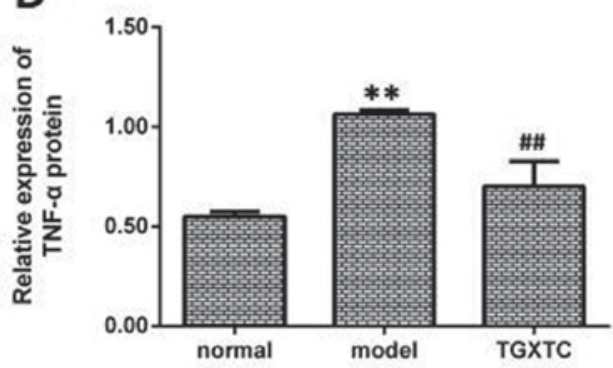

Figure 8. Effects of TGXTC on the protein expression of IL-1 $\beta$ and TNF- $\alpha$. The protein expression of IL-1 $\beta$ and TNF- $\alpha$ was determined by western blot analysis. $\beta$-actin was used as the internal control. (A and C) and (B and D) Representative images are shown. Data shown represent the mean \pm standard deviation. ${ }^{* *} \mathrm{P}<0.01$ vs. normal group; ${ }^{\# \#} \mathrm{P}<0.01$ vs. model group. TGXTC, Tougu Xiaotong capsule.

TGXTC decreased the OPG/RANKL ratio. The ratio of $\mathrm{OPG} / \mathrm{RANKL}$ was significantly higher in the model than that in the normal group $(\mathrm{P}<0.01$; Fig. $6 \mathrm{C})$. After treatment, the ratio of OPG/RANKL was significantly decreased in the TGXTC group compared to the model group $(\mathrm{P}<0.01$; Fig. $6 \mathrm{C})$.

TGXTC inhibits the mRNA expression of $I L-1 \beta$ and TNF- $\alpha$. The mRNA expression of IL-1 $\beta$ was significantly higher in the model than that in the normal group $(\mathrm{P}<0.01$; Fig. 7A). After treatment, the IL-1 $\beta$ mRNA expression was significantly decreased in the TGXTC group compared with the model group $(\mathrm{P}<0.01$; Fig. 7A). mRNA expression of TNF- $\alpha$ was also significantly higher in the model compared to the normal group $(\mathrm{P}<0.01$; Fig. 7B). After treatment, TNF- $\alpha$ mRNA expression was also significantly decreased in the TGXTC group compared with the model group $(\mathrm{P}<0.01$; Fig. 7B).

TGXTC inhibits the protein expression of $I L-1 \beta$ and TNF- $\alpha$. The protein expression of IL-1 $\beta$ was significantly higher in the model than the normal group $(\mathrm{P}<0.01$; Fig. 8A and $\mathrm{C})$. After treatment, the IL-1 $\beta$ protein expression was significantly decreased in the TGXTC compared with the model group
$(\mathrm{P}<0.01$; Fig. 8A and C). The protein expression of TNF- $\alpha$ was significantly higher in the model than the normal group $(\mathrm{P}<0.01$; Fig. 8B and D). After treatment, the TNF- $\alpha$ protein expression was markedly decreased in the TGXTC compared with the model group $(\mathrm{P}<0.01$; Fig. $8 \mathrm{~B}$ and $\mathrm{D})$.

\section{Discussion}

Although significant progress has been made in understanding OA pathophysiological pathways, much remains to be done to develop a specific therapy that could effectively retard or prevent progression of the disease. To this end, the literature suggests that in addition to cartilage, other articular tissues, including the subchondral bone, should be targeted. Previous findings have shown that TGXTC has therapeutic effects on knee OA (25) through multiple targets $(22,23,25-27)$. In this study, we investigated the effect of TGXTC on subchondral bone and its relevant mechanisms of inflammatory factors.

To determine the effects of TGXTC on cartilage structure and the stage of OA, we used the Mankin score. In the model group, the articular cartilage structure was damaged; the stained proteoglycans became weaker, showing severe loss 
of proteoglycans. However, TGXTC treatment altered loss of proteoglycans and appearance of the cartilage structure, and the proteoglycan staining was improved compared to the normal group. The result shows that TGXTC improves cartilage matrix to promote increased production of proteoglycans. Additionally, the lower the modified Mankin score of the animal is, the better the outcome with respect to delaying the progression of OA (9). Therefore, we used Mankin scoring principles to analyze alteration of cartilage degeneration after TGXTC treatment. We found the Mankin score was decreased in the TGXTC group compared with the model group, which showed the stage of OA is from the middle stage to the early stage. These findings suggest that TGXTC has better therapeutic effects on mitigating cartilage damage and preventing the development of OA.

As the subchondral bone and cartilage are closely related, structural changes in the subchondral bone can further aggravate cartilage damage. Thus, we can delay the progression of OA by regulating bone remodeling to improve the subchondral bone structure $(30,31)$. In the present study, the effect of TGXTC on subchondral bone was observed by micro-CT, which enables non-destructive assessment of the subchondral bone both quantitatively and qualitatively (10). We found osteophyte formation in the model group, with osteophytes narrowing the area in TGXTC group. In the model group, the increase of Tb.N and BV/TV indicate subchondral bone trabeculae undergo sclerosis. However, after treatment, the sclerosis was improved. We speculate the treatment with TGXTC could change these parameters by regulating bone remodeling, and the structure of subchondral bone was altered by improving the trabecular bone parameters.

Bone remodeling is a highly complex process by which old bone is replaced by new bone, in a cycle comprised of three phases: i) Initiation of bone resorption by osteoclasts, ii) the transition (or reversal period) from resorption to new bone formation, and iii) the bone formation by osteoblasts $(32,33)$. ALP is essential for bone mineralization, which is secreted by osteoblast (34). Increased ALP level in serum has been observed in conditions such as rapid bone loss (35) and fracture risk $(36,37)$. TRAP is secreted by osteoclasts during bone resorption, and serum TRAP activity correlates with resorptive activity in disorders of bone metabolism (14). In order to further explore the regulatory mechanism of the subchondral bone reconstruction, ALP and TRAP activity in serum was detected. In the present study a significant increase in ALP and TRAP levels was observed in the model group. These results indicated that a high bone formation and bone resorption of the bone remodeling process were both increased in OA. Subchondral sclerosis was observed using micro-CT; thus, we suggest that the rate of bone formation is greater than the rate of bone resorption. By contrast, treatment of TGXTC showed significantly decreased ALP and TRAP activity, suggesting that the potency of TGXTC is due to a decrease in the bone remodeling process by reducing bone formation and resorption to alleviate subchondral bone sclerosis in OA rabbits.

During OA, bone remodeling causes an imbalance between bone resorption and bone formation, leading to structural changes in the subchondral bone. Recently, the OPG/RANKL signalling pathway was shown to be involved in the regulation of osteoblastogenesis and osteoblast activity, which was regarded as a key factor in inhibiting bone proliferation and directly participates in the process of bone formation and bone resorption $(38,39)$. Bone remodeling is also controlled by the balance of the OPG/RANKL ratio $(18,40)$. Higher OPG/RANKL ratios mediate bone formation (41), while lower OPG/RANKL ratios mediate bone resorption (42). The OPG/RANKL production ratio was significantly increased in the model group, indicating that the remodeling rate of the subchondral bone was faster, excessive bone protection developed, and bone resorption was suppressed. However, following treatment with TGXTC, the OPG/RANKL ratio was significantly decreased, suggesting that TGXTC can reduce the remodeling rate by stabilizing bone resorption and bone formation to delay subchondral bone damage.

Previous studies proved that the regulation of bone metabolism occurs through the classical OPG/RANK/RANKL pathway, the direct and indirect functions of which are influenced by inflammatory factors, such as IL-1 $\beta$, TNF- $\alpha$, IL-6 and IL-17 (20,22,43-48). There are reports that TNF- $\alpha$ and IL-1 $\beta$ and other inflammatory factors can reduce OPG, stimulate RANKL and have a synergistic effect, resulting in increased bone resorption $(47,49)$. In the present study, we found that the mRNA and protein expression of IL-1 and TNF- $\alpha$ in subchondral bone initially exhibited a significant increase in the model group and then decreased after treatment with TGXTC. These results indicated that inflammatory factors increased in OA, and TGXTC can decrease inflammatory factors to improve joint damage.

Taken together, results of the present study suggest that TGXTC could delay the pathological development of OA by regulating subchondral bone remodeling through regulation of the formation/resorption balance and its relating inflammatory factors. This may partly explain its clinical efficacy in the treatment of knee OA.

\section{Acknowledgements}

The authors would like to thank Professor Liu Xianxiang, from the Academy of Integrative Medicine, Fujian University of Traditional Chinese Medicine, for his helpful discussion.

\section{Funding}

This study was supported by the National Natural Science Foundation of China (grant no. 81774345) and the Developmental Fund of Chen Keji Integrative Medicine (grant no. CKJ2018003).

\section{Availability of data and materials}

The datasets used during the present study are available from the corresponding author on reasonable request.

\section{Authors' contributions}

WC and SC conceived and designed the study, and reviewed and edited the manuscript. $\mathrm{YH}$ and $\mathrm{MH}$ performed the histology experiment. RL and ZL performed the micro-CT evaluation experiment. LZ performed the serum biochemistry assay experiment. JZ and GW performed the RT-qPCR experiment 
and the data analysis. XL and SC performed the western blot analysis experiment. GW designed the experiment and wrote the manuscript. All authors approved the final version of the manuscript.

\section{Ethics approval and consent to participate}

The present study was approved by the Animal Care and Use Committee of Fujian University of Traditional Chinese Medicine (Fujian, China).

\section{Patient consent for publication}

Not applicable.

\section{Competing interests}

The authors declare that they have no competing interests.

\section{References}

1. Mansell JP, Tarlton JF and Bailey AJ: Biochemical evidence for altered subchondral bone collagen metabolism in osteoarthritis of the hip. Br J Rheumatol 36: 16-19, 1997.

2. Bobinac D, Marinovic M, Bazdulj E, Cvijanovic O, Celic T, Maric I, Spanjol J and Cicvaric T: Microstructural alterations of femoral head articular cartilage and subchondral bone in osteoarthritis and osteoporosis. Osteoarthritis Cartilage 21: $1724-1730,2013$

3. Kwan Tat S, Pelletier JP, Lajeunesse D, Fahmi H, Lavigne M and Martel-Pelletier J: The differential expression of osteoprotegerin (OPG) and receptor activator of nuclear factor kappaB ligand (RANKL) in human osteoarthritic subchondral bone osteoblasts is an indicator of the metabolic state of these disease cells. Clin Exp Rheumatol 26: 295-304, 2008.

4. Tat SK, Pelletier JP, Lajeunesse D, Fahmi H, Duval N and Martel-Pelletier J: Differential modulation of RANKL isoforms by human osteoarthritic subchondral bone osteoblasts: Influence of osteotropic factors. Bone 43: 284-291, 2008.

5. Orth P, Cucchiarini M, Kaul G, Ong MF, Gräber S, Kohn DM and Madry $\mathrm{H}$ : Temporal and spatial migration pattern of the subchondral bone plate in a rabbit osteochondral defect model. Osteoarthritis Cartilage 20: 1161-1169, 2012.

6. Roman-Blas JA and Herrero-Beaumont G: Targeting subchondral bone in osteoporotic osteoarthritis. Arthritis Res Ther 16: 494 2014.

7. Li G, Yin J, Gao J, Cheng TS, Pavlos NJ, Zhang C and Zheng MH: Subchondral bone in osteoarthritis: Insight into risk factors and microstructural changes. Arthritis Res Ther 15: 223, 2013.

8. Effendy NM, Khamis MF and Shuid AN: The effects of Labisia pumila extracts on bone microarchitecture of ovariectomizedinduced osteoporosis rats: A micro-CT analysis. J XRay Sci Technol 25: 101-112, 2017.

9. Permuy M, Guede D, López-Peña M, Muñoz F, Caeiro JR and González-Cantalapiedra A: Effects of diacerein on cartilage and subchondral bone in early stages of osteoarthritis in a rabbit model. BMC Vet Res 11: 143, 2015.

10. Mohan G, Perilli E, Kuliwaba JS, Humphries JM, Parkinson IH and Fazzalari NL: Application of in vivo micro-computed tomography in the temporal characterisation of subchondral bone architecture in a rat model of low-dose monosodium iodoacetateinduced osteoarthritis. Arthritis Res Ther 13: R210, 2011.

11. Bellido M, Lugo L, Roman-Blas JA, Castañeda S, Caeiro JR, Dapia S, Calvo E, Largo R and Herrero-Beaumont G: Subchondral bone microstructural damage by increased remodelling aggravates experimental osteoarthritis preceded by osteoporosis. Arthritis Res Ther 12: R152, 2010.

12. Zhou J, Ma XN, Gao YH, Yan JL, Shi WG, Xian CJ and Chen KM: Sinusoidal electromagnetic fields promote bone formation and inhibit bone resorption in rat femoral tissues in vitro. Electromagn Biol Med 35: 75-83, 2016.

13. Huang LQ, He HC, He CQ, Chen J and Yang L: Clinical update of pulsed electromagnetic fields on osteoporosis. Chin Med J (Engl) 121: 2095-2099, 2008
14. Janckila AJ and Yam LT: Biology and clinical significance of tartrate-resistant acid phosphatases: New perspectives on an old enzyme. Calcif Tissue Int 85: 465-483, 2009.

15. Suchecki D and Tufik S: Social stability attenuates the stress in the modified multiple platform method for paradoxical sleep deprivation in the rat. Physiol Behav 68: 309-316, 2000.

16. Bolon B, Grisanti M, Villasenor K, Morony S, Feige U and Simonet WS: Generalized Degenerative Joint Disease in Osteoprotegerin (Opg) Null Mutant Mice. Vet Pathol 52: 873-882, 2015.

17. Shen Y, Jiang T, Wang R, He S, Guo M, Zuo J and He D: (5R)-5Hydroxytriptolide (LLDT-8) inhibits osteoclastogenesis via RANKL/RANK/OPG signaling pathway. BMC Complement Altern Med 15: 77, 2015.

18. Trouvin AP and Goëb V: Receptor activator of nuclear factor- $\kappa B$ ligand and osteoprotegerin: Maintaining the balance to prevent bone loss. Clin Interv Aging 5: 345-354, 2010.

19. Lange U, Dischereit G, Tarner I, Frommer K, Neumann E, Müller-Ladner U and Kürten B: The impact of serial radon and hyperthermia exposure in a therapeutic adit on pivotal cytokines of bone metabolism in rheumatoid arthritis and osteoarthritis. Clin Rheumatol 35: 2783-2788, 2016.

20. Aida Y, Maeno M, Suzuki N, Shiratsuchi H, Motohashi M and Matsumura $\mathrm{H}$ : The effect of IL-1beta on the expression of matrix metalloproteinases and tissue inhibitors of matrix metalloproteinases in human chondrocytes. Life Sci 77: 3210-3221, 2005.

21. Teitelbaum SL: Bone resorption by osteoclasts. Science 289: 1504-1508, 2000.

22. Liao N, Huang Y, Ye J, Chen W, Li ZF, Lin R, Li X, Zheng L and Liu X: Protective effects of Tougu Xiaotong capsule on tumor necrosis factor- $\alpha$-injured UMR-106 cells. Exp Ther Med 10: 1908-1914, 2015.

23. Chen S, Huang Y, Chen W, Wu G, Liao N, Li X, Huang M, Lin R, Yu C, Li X, et al: Protective effects of the Tougu Xiaotong capsule on morphology and osteoprotegerin/nuclear factor- $\kappa \mathrm{B}$ ligand expression in rabbits with knee osteoarthritis. Mol Med Rep 13: 419-425, 2016.

24. Zheng CS, Ye HZ, Xu XJ and Liu XX: Computational pharmacology study of tougu xiaotong granule in preventing and treating knee osteoarthritis. Chin J Integr Med 15: 371-376, 2009.

25. Lin MN and Liu XX: Observation of Tougu Xiaotong Recipe for the treatment of 30 patients with knee osteoarthritis. Fujian J Tradit Chin Med Chin 36: 15-16, 2005 (In Chinese).

26. Li XH, Wu MX, Ye HZ, Chen WL, Lin JM, Zheng LP and Liu XX: Experimental study on the suppression of sodium nitroprussiate-induced chondrocyte apoptosis by Tougu Xiaotong Capsule -containing serum. Chin J Integr Med 17: 436-443, 2011.

27. Li X, Lang W, Ye H, Yu F, Li H, Chen J, Cai L, Chen W, Lin R, Huang Y, et al: Tougu Xiaotong capsule inhibits the tidemark replication and cartilage degradation of papain-induced osteoarthritis by the regulation of chondrocyte autophagy. Int J Mol Med 31: 1349-1356, 2013.

28. Liu XX, Li XH and Zhou JT: [Experimental study on replicating knee osteoarthritis by modified Hulth's modeling method]. Zhongguo Zhong Xi Yi Jie He Za Zhi 25: 1104-1108, 2005 (In Chinese).

29. Mankin HJ, Dorfman H, Lippiello L and Zarins A: Biochemical and metabolic abnormalities in articular cartilage from osteoarthritic human hips. II. Correlation of morphology with biochemical and metabolic data. J Bone Joint Surg Am 53: 523-537, 1971.

30. Cox LG, van Donkelaar CC, van Rietbergen B, Emans PJ and Ito K: Decreased bone tissue mineralization can partly explain subchondral sclerosis observed in osteoarthritis. Bone 50: 1152-1161, 2012.

31. Zhu S, Chen K, Lan Y, Zhang N, Jiang R and Hu J: Alendronate protects against articular cartilage erosion by inhibiting subchondral bone loss in ovariectomized rats. Bone 53: 340-349, 2013.

32. Geusens PP and van den Bergh JP: Osteoporosis and osteoarthritis: Shared mechanisms and epidemiology. Curr Opin Rheumatol 28: 97-103, 2016.

33. Matsuo $\mathrm{K}$ and Irie N: Osteoclast-osteoblast communication. Arch Biochem Biophys 473: 201-209, 2008.

34. Havill LM, Hale LG, Newman DE, Witte SM and Mahaney MC: Bone ALP and OC reference standards in adult baboons (Papio hamadryas) by sex and age. J Med Primatol 35: 97-105, 2006

35. Ni J, Yuan XM, Yao Q and Peng LB: OSM is overexpressed in knee osteoarthritis and Notch signaling is involved in the effects of OSM on MC3T3-E1 cell proliferation and differentiation. Int J Mol Med 35: 1755-1760, 2015. 
36. Bellido M, Lugo L, Roman-Blas JA, Castañeda S, Calvo E, Largo R and Herrero-Beaumont G: Improving subchondral bone integrity reduces progression of cartilage damage in experimental osteoarthritis preceded by osteoporosis. Osteoarthritis Cartilage 19: 1228-1236, 2011.

37. Ross PD, Kress BC, Parson RE, Wasnich RD, Armour KA and Mizrahi IA: Serum bone alkaline phosphatase and calcaneus bone density predict fractures: A prospective study. Osteoporos Int 11: 76-82, 2000

38. Kong YY, Yoshida H, Sarosi I, Tan HL, Timms E, Capparelli C, Morony S, Oliveira-dos-Santos AJ, Van G, Itie A, et al: OPGL is a key regulator of osteoclastogenesis, lymphocyte development and lymph-node organogenesis. Nature 397: 315-323, 1999.

39. Hofbauer LC and Schoppet M: Clinical implications of the osteoprotegerin/RANKL/RANK system for bone and vascular diseases. JAMA 292: 490-495, 2004.

40. Whyte MP, Obrecht SE, Finnegan PM, Jones JL, Podgornik MN, McAlister WH and Mumm S: Osteoprotegerin deficiency and juvenile Paget's disease. N Engl J Med 347: 175-184, 2002.

41. Brzóska MM and Rogalska J: Protective effect of zinc supplementation against cadmium-induced oxidative stress and the RANK/RANKL/OPG system imbalance in the bone tissue of rats. Toxicol Appl Pharmacol 272: 208-220, 2013.

42. Gurban CV and Mederle O: The OPG/RANKL system and zinc ions are promoters of bone remodeling by osteoblast proliferation in postmenopausal osteoporosis. Rom J Morphol Embryol 52: $1113-1119,2011$.

43. FujiiT,KitauraH,KimuraK,HakamiZWandTakano-YamamotoT: IL-4 inhibits TNF- $\alpha$-mediated osteoclast formation by inhibition of RANKL expression in TNF- $\alpha$-activated stromal cells and direct inhibition of TNF- $\alpha$-activated osteoclast precursors via a T-cell-independent mechanism in vivo. Bone 51: 771-780, 2012.
44. Onal M, Xiong J, Chen X, Thostenson JD, Almeida M, Manolagas SC and O'Brien CA: Receptor activator of nuclear factor $\chi \mathrm{B}$ ligand (RANKL) protein expression by $\mathrm{B}$ lymphocytes contributes to ovariectomy-induced bone loss. J Biol Chem 287: 29851-29860, 2012.

45. Riegel A, Maurer T, Prior B, Stegmaier S, Heppert V, Wagner C and Hänsch GM: Human polymorphonuclear neutrophils express RANK and are activated by its ligand, RANKL. Eur J Immunol 42: 975-981, 2012.

46. Kim KW, Kim HR, Park JY, Park JS, Oh HJ, Woo YJ, Park MK, Cho ML and Lee SH: Interleukin-22 promotes osteoclastogenesis in rheumatoid arthritis through induction of RANKL in human synovial fibroblasts. Arthritis Rheum 64: 1015-1023, 2012.

47. Lam J, Takeshita S, Barker JE, Kanagawa O, Ross FP and Teitelbaum SL: TNF-alpha induces osteoclastogenesis by direct stimulation of macrophages exposed to permissive levels of RANK ligand. J Clin Invest 106: 1481-1488, 2000.

48. Onal M, Galli C, Fu Q, Xiong J, Weinstein RS, Manolagas SC and O'Brien CA: The RANKL distal control region is required for the increase in RANKL expression, but not the bone loss, associated with hyperparathyroidism or lactation in adult mice. Mol Endocrinol 26: 341-348, 2012.

49. Zeng JZ, Ma LF, Meng H, Yu HM, Zhang YK and Guo A: (5R)-5-hydroxytriptolide (LLDT-8) prevents collagen-induced arthritis through OPG/RANK/RANKL signaling in a rat model of rheumatoid arthritis. Exp Ther Med 12: 3101-3106, 2016. 